\section{Multiple aortic aneurysms because of infective endocarditis after repair of aortic coarctation}

Infective endocarditis (IE) is a rare disease in the pediatric population, and its annual incidence rate in the United States was approximately between 0.05 and 0.12 cases per 1,000 pediatric admissions according to the last pediatric American Heart Association guidelines (1). Although aneurysms with abnormal focal arterial enlargement are rare both in the ascending and the descending aorta, they are known to occur in patients with aortic coarctation with or without a history of surgical repair $(2,3)$.

A 6-month-old-boy was referred to our clinic owing to recoarctation of aorta ( $\left.C_{0} A\right)$. His medical history revealed that he underwent a surgical repair of the CoA using an extended endto-end method with lateral thoracotomy in his first month of life. After successful balloon angioplasty in the early postoperative period, he was discharged from the hospital uneventfully. $\mathrm{He}$ was referred to our center again with the diagnosis of IE owing to a major embolic event in the second month of his follow-up. Two-dimensional transthoracic echocardiography revealed severe aortic regurgitation, aortic cusp prolapsus, bicuspid aortic leaflet thickening, and severe re-CoA at the descending aorta. There were also mobile vegetation and multiple pseudoaneurysms in the aorta. His blood cultures (48 hours apart) were

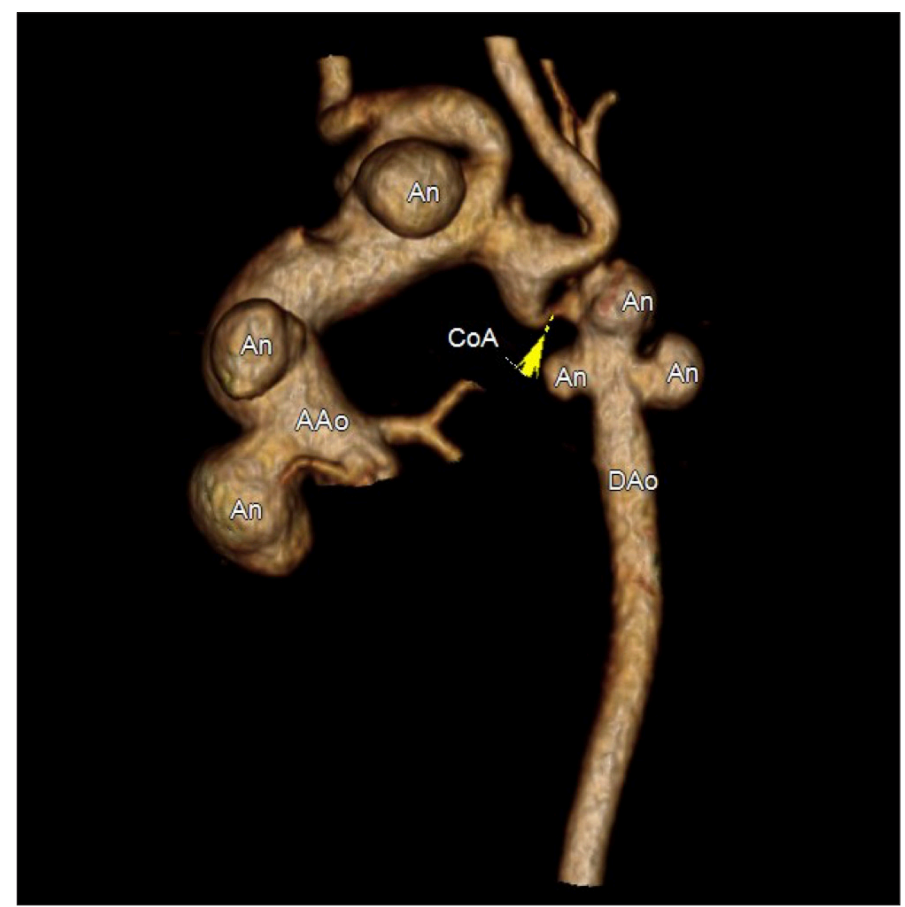

Figure 1. Computed tomography angiography showing saccular aneurysms and re-coarctation of aorta

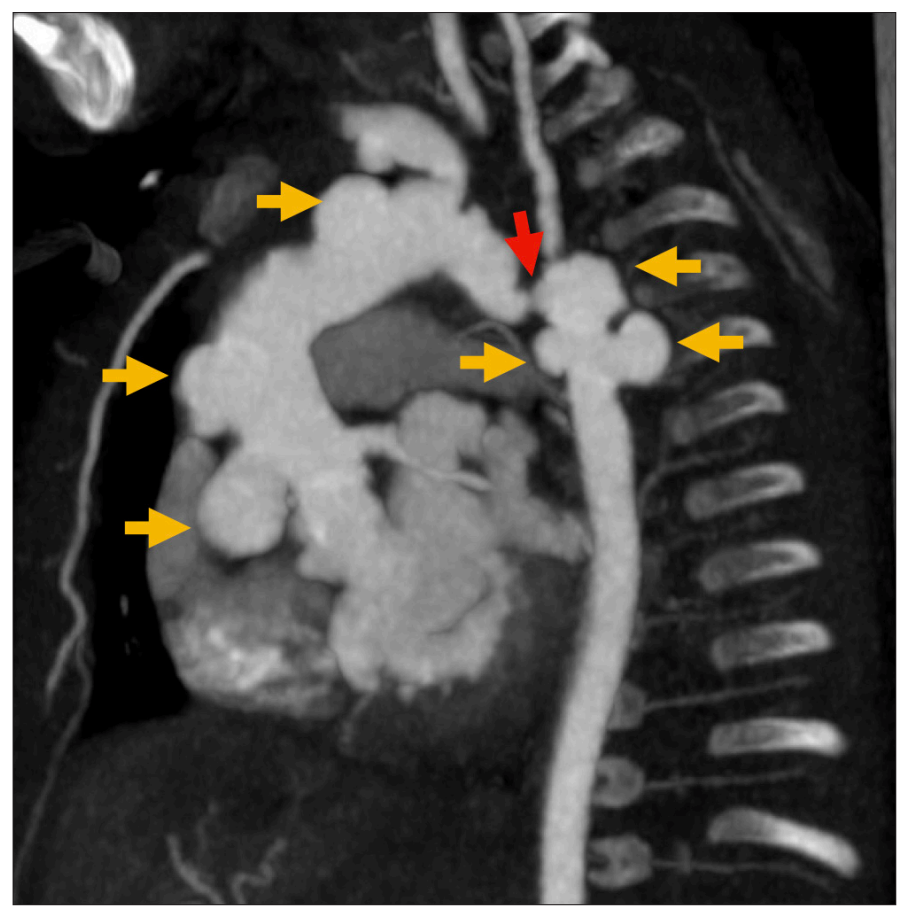

Figure 2. Computed tomography angiography showing saccular aneurysms (yellow arrows) and re-coarctation (red arrow) of aorta

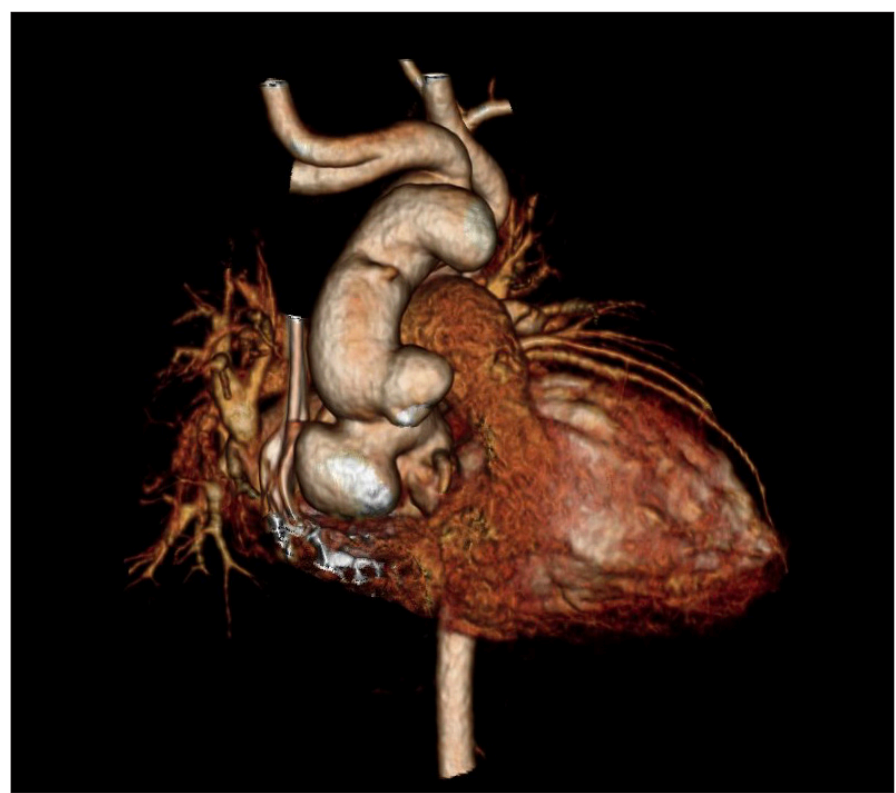

Figure 3. Computed tomography angiography demonstrating saccular aneurysms in the ascending aorta

observed to contain Staphylococcus epidermidis, and he was diagnosed with IE according to the modified Duke criteria.

Computed tomography angiography (CTA) showed multiple aneurysms in different parts of the ascending and descending aorta and a stenosis of the distal part of the aortic arch and recoarctation (Fig. 1, 2). CTA demonstrated the first saccular aneurysm with a diameter of $13 \times 18 \mathrm{~mm}$ located $5 \mathrm{~mm}$ superior to the 
level of the sinotubular junction of the ascending aorta. The second aneurysm with a diameter of $11 \times 12 \mathrm{~mm}$ was located 14 $\mathrm{mm}$ superior to the sinotubular level of the ascending aorta, and the third aneurysm with a diameter of $13 \times 14 \mathrm{~mm}$ at the anterior level of the distal part of the ascending aorta (Fig. 3). CTA also showed the remaining 3 aneurysms in the descending aorta. All of them originated from the proximal part of the descending aorta with diameters of $5 \times 6,9 \times 10$, and $11 \times 11 \mathrm{~mm}$, respectively (Video 1).

Surgery was planned after a 4-week treatment with vancomycin, gentamicin, fluconazole, and rifampicin.

Informed consent: Written informed consent was obtained from the parents.

Video 1. Computed tomography angiography demonstrating multiple saccular aneurysms in the ascending and descending aorta

\section{Reference}

1. Baltimore RS, Gewitz M, Baddour LM, Beerman LB, Jackson MA, Lockhart PB, et al.; American Heart Association Rheumatic Fever, Endocarditis, and Kawasaki Disease Committee of the Council on Cardiovascular Disease in the Young and the Council on Cardiovascular and Stroke Nursing. Infective Endocarditis in Childhood: 2015 Update: A Scientific Statement From the
American Heart Association. Circulation 2015; 132: 1487-515. [Crossref]

2. Restrepo MS, Turek JW, Reinking B, Bergen NV. Mycotic aneurysm in a child with history of coarctation of the aorta repair. Ann Pediatr Cardiol 2014; 7: 138-41. [Crossref]

3. Ratsimandresy M, Hadeed K, Acar P, Cuttone F. A case report of mycotic pseudoaneurysm in childhood: an unusual complication of coarctation of the aorta. Eur Heart J Case Rep 2019; 3: yty170.

\section{Erman Çilsal* (D), Bahar Çaran* (D), Aysel Türkvatan** (D), Hacer Kamalı* (D), Sertaç Haydin* (D), Alper Güzeltaş* (D)}

Departments of *Pediatric Cardiology, and **Radiology, University of Health Sciences, Mehmet Akif Ersoy Thoracic and Cardiovascular Surgery Training and Research Hospital; İstanbulTurkey

Address for Correspondence: Dr. Erman Çilsal, Mehmet Akif Ersoy Göğüs Kalp ve Damar Cerrahisi Eğitim ve Araştırma Hastanesi, Çocuk Kardiyoloji Kliniği, İstanbul-Türkiye Phone: +90 21269220 00/1157

E-mail: ermancilsal@gmail.com

CCopyright 2021 by Turkish Society of Cardiology Available online at www. anatoljcardiol.com DOI:10.5152/AnatolJCardiol.2021.350 